Supplement of Atmos. Chem. Phys., 22, 2651-2668, 2022

https://doi.org/10.5194/acp-22-2651-2022-supplement

(C) Author(s) 2022. CC BY 4.0 License.

(c) (1)

Atmospheric
Chemistry
and Physics

Supplement of

\title{
First observation of mercury species on an important water vapor channel in the southeastern Tibetan Plateau
}

Huiming Lin et al.

Correspondence to: Yindong Tong (yindongtong@tju.edu.cn) and Xuejun Wang (wangxuejun@pku.edu.cn)

The copyright of individual parts of the supplement might differ from the article licence. 


\section{Figure captions}

Figure S1 Indian Monsoon Index in 2019 (data from cmdp.ncc-cma.net)

Figure S2 Species Hg monitoring in Nyingchi

Figure S3 Trajectories to Nyingchi in June 2019 with different arrival height

Figure S4 Trajectories to Nyingchi for difference seasons (March ,April and May (MAM),June, July and August (JJA),September, October and November (SON), December, January and February (DJF))

Figure S5 Trajectories to Nyingchi with high PAS monitoring period and low PAS monitoring period

\section{Table captions}

Table S1 start time and finish time of passive samplers at SET station

Table S2 Statistical metrics of species Hg, meteorological factors, and other pollutants

Table S3 daily average concentrations of $\mathrm{Hg}$ species, meteorological and some pollutants from 31 May to 3 June, 2019. Red markers indicate large changes in factors 
Figure S1 Indian Monsoon Index in 2019 (data from cmdp.ncc-cma.net)

Indian Monsoon Index 2019

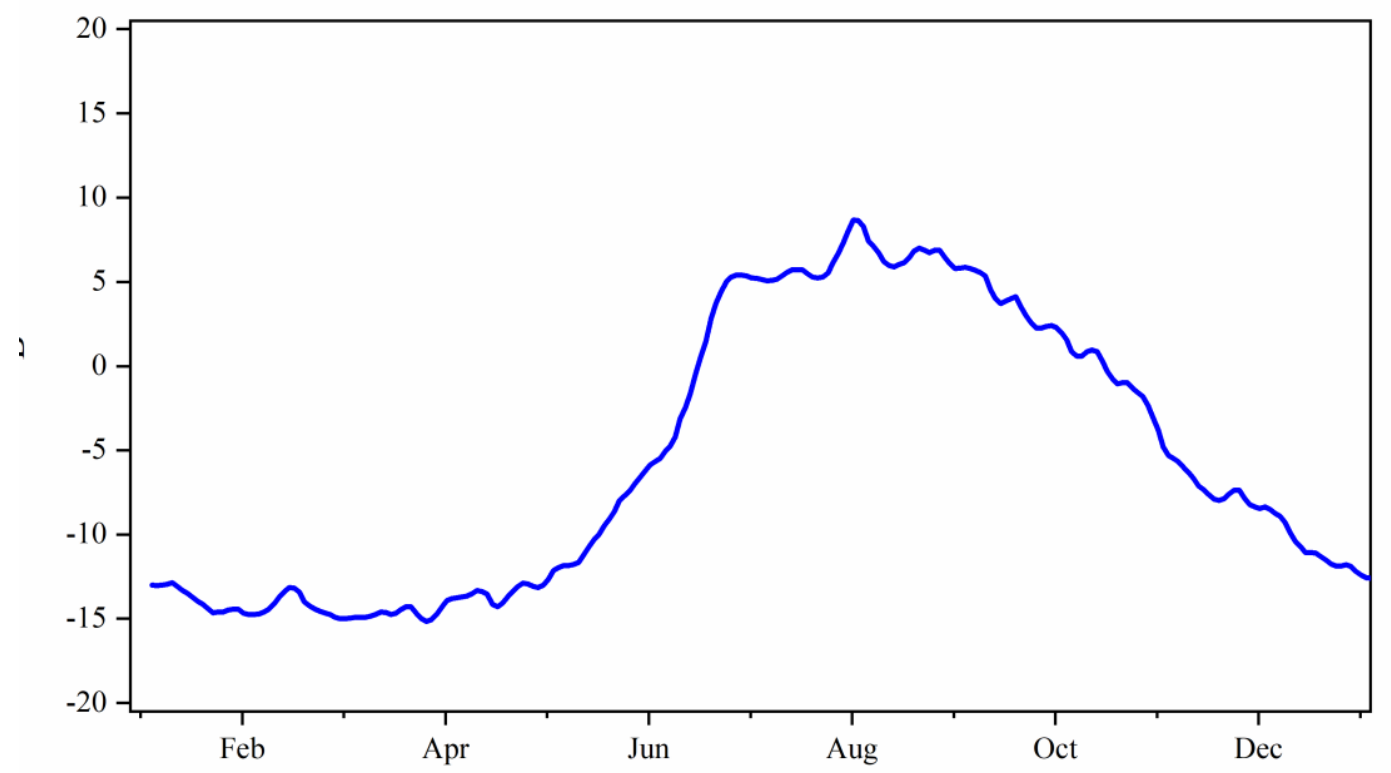


Figure S2 Species Hg monitoring in Nyingchi

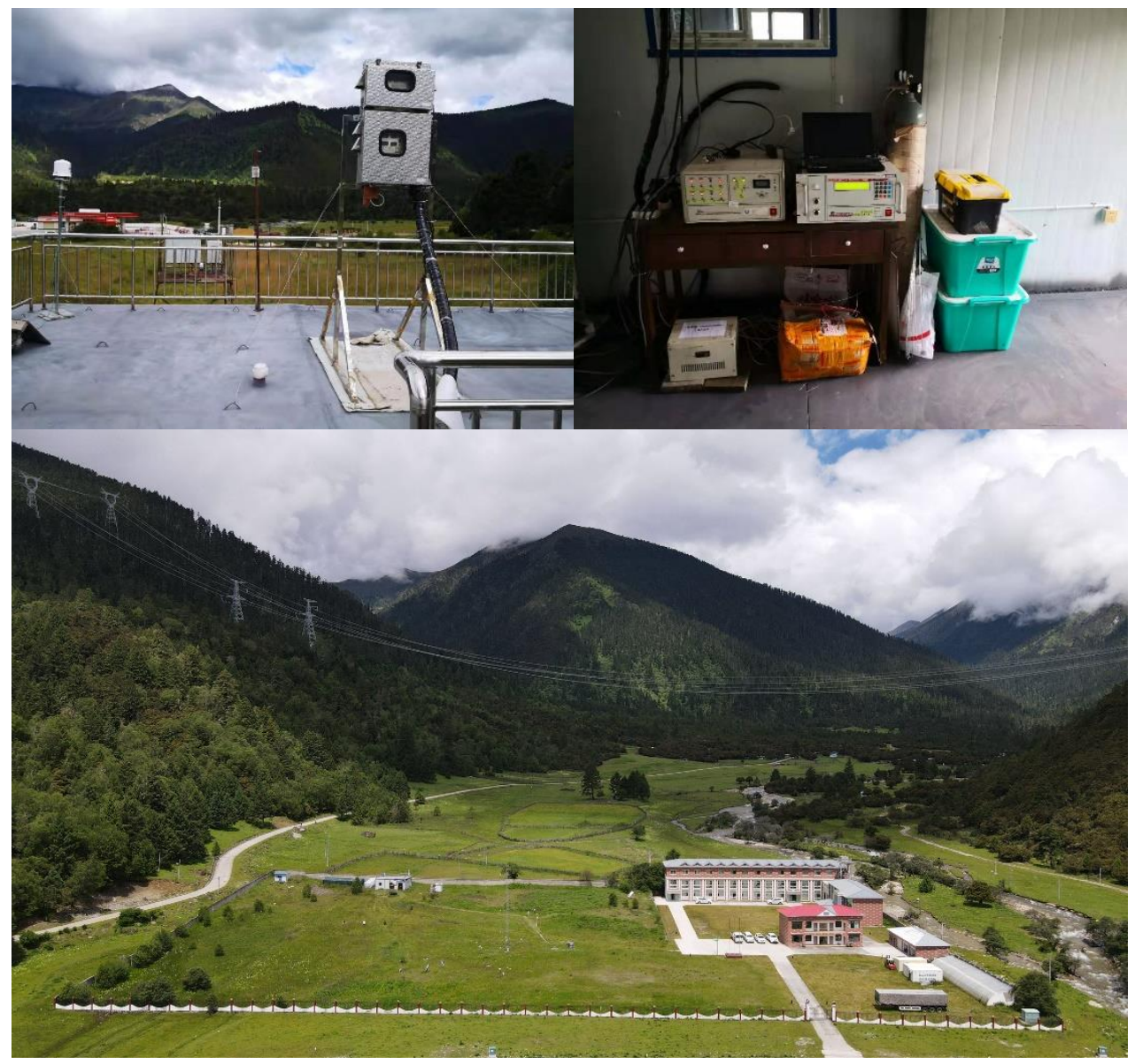


Figure S3 Trajectories to Nyingchi in June 2019 with different arrival height

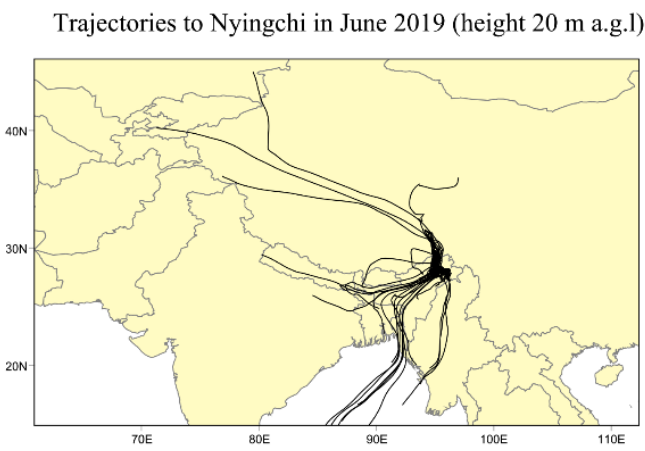

Trajectories to Nyingchi in June 2019 (height $50 \mathrm{~m}$ a.g.l)

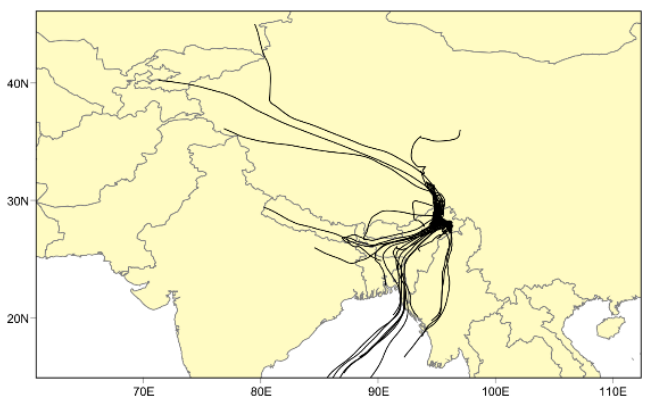

Trajectories to Nyingchi in June 2019 (height $200 \mathrm{~m}$ a.g.l)

Trajectories to Nyingchi in June 2019 (height $500 \mathrm{~m}$ a.g.1)
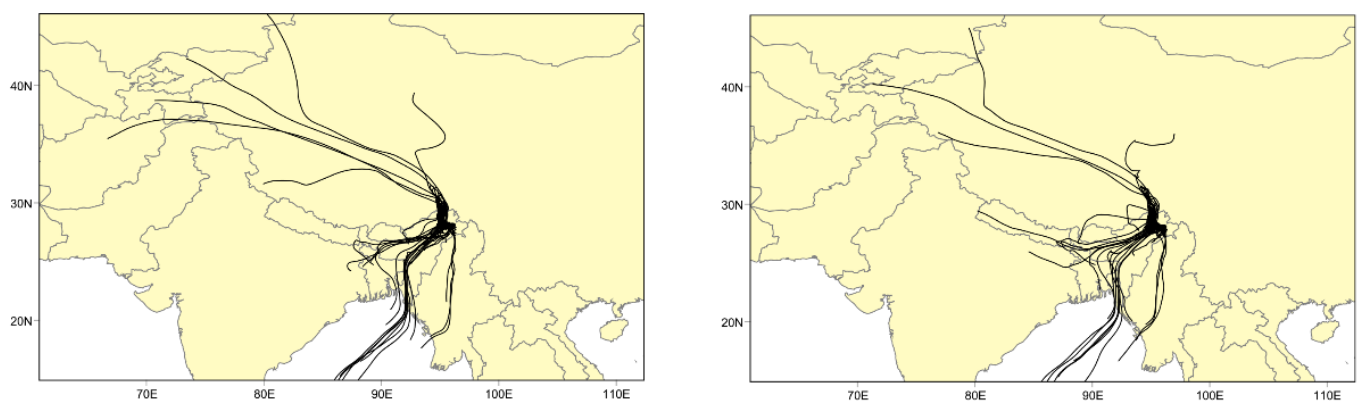
Figure S4 Trajectories to Nyingchi for difference seasons (March ,April and May (MAM),June, July and August (JJA),September, October and November (SON), December, January and February (DJF))
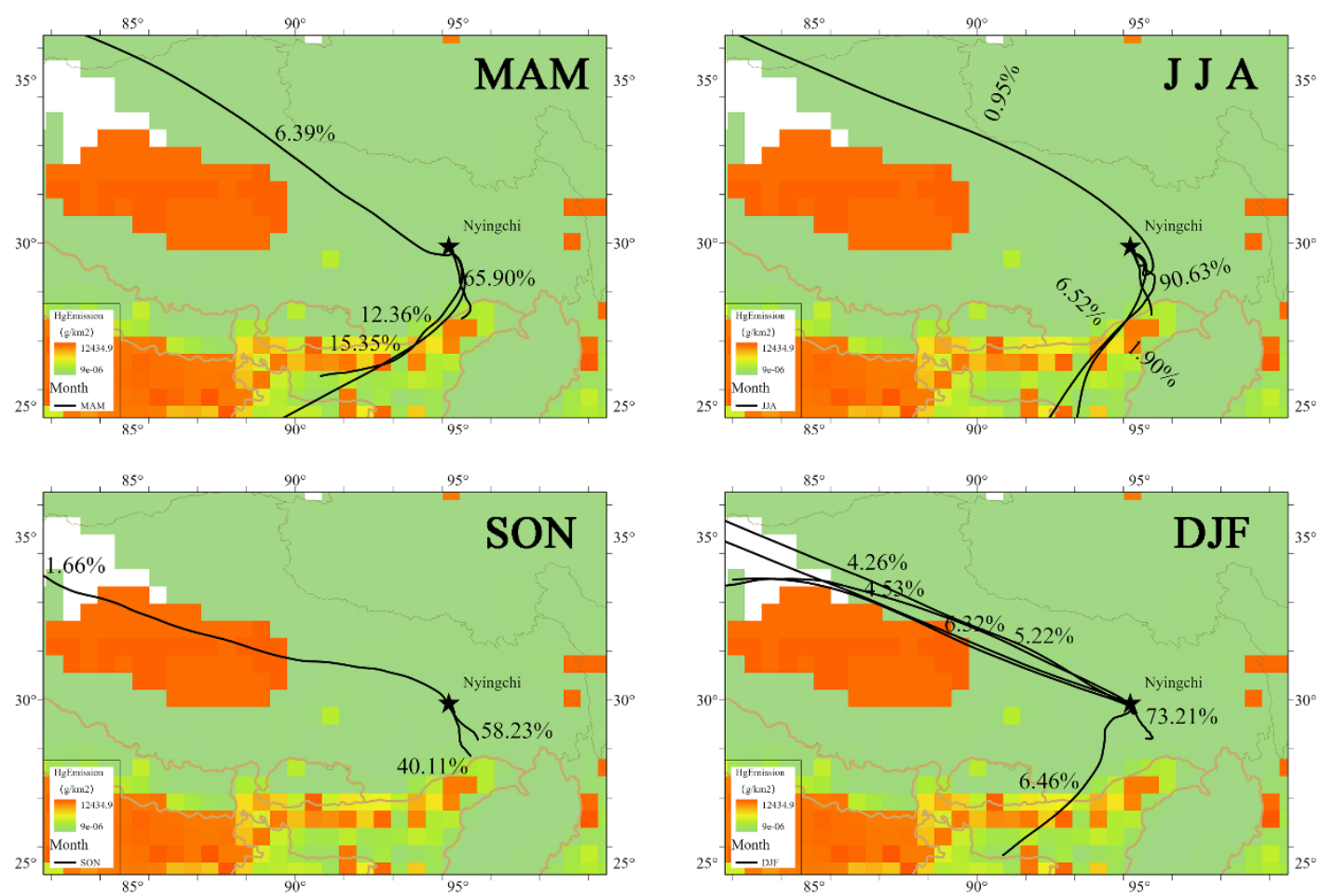
Figure S5 Trajectories to Nyingchi with high PAS monitoring period and low PAS monitoring period

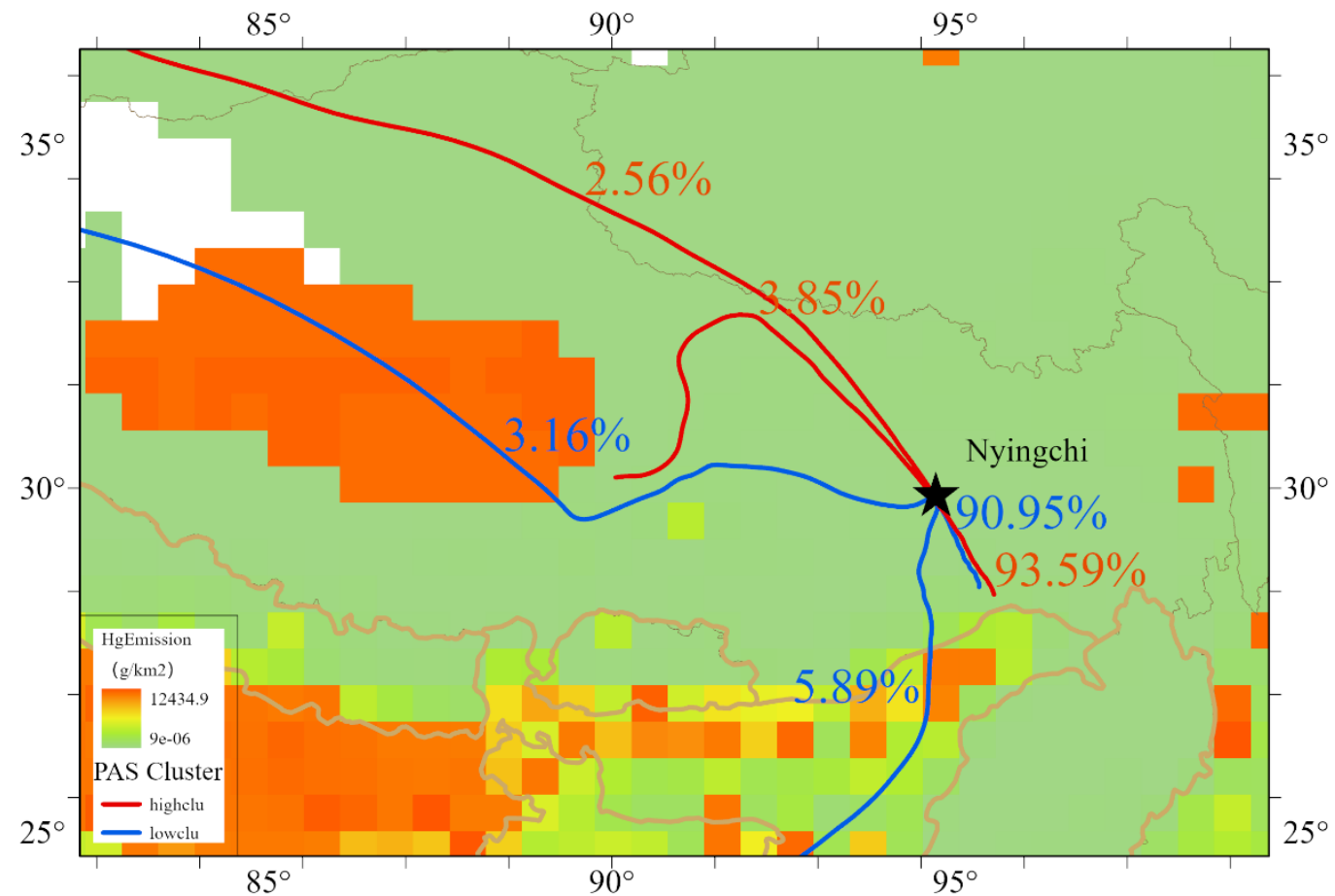


Table S1 start time and finish time of passive samplers at SET station

\begin{tabular}{cc}
\hline start time & finish time \\
\hline $2019 / 4 / 412: 00$ & $2019 / 5 / 112: 00$ \\
$2019 / 5 / 112: 00$ & $2019 / 6 / 913: 15$ \\
2019/6/9 13:15 & $2019 / 7 / 1013: 15$ \\
$2019 / 7 / 1013: 15$ & $2019 / 7 / 2119: 30$ \\
$2019 / 7 / 2119: 30$ & $2019 / 8 / 113: 00$ \\
2019/8/1 13:00 & $2019 / 8 / 713: 00$ \\
2019/8/7 13:00 & $2019 / 8 / 2113: 40$ \\
$2019 / 8 / 2113: 40$ & $2019 / 9 / 115: 50$ \\
$2019 / 9 / 115: 50$ & $2019 / 9 / 1112: 10$ \\
$2019 / 9 / 1112: 10$ & $2019 / 9 / 2116: 30$ \\
$2019 / 9 / 2116: 30$ & $2019 / 10 / 117: 00$ \\
$2019 / 10 / 117: 00$ & $2019 / 10 / 1210: 00$ \\
$2019 / 10 / 1210: 00$ & $2019 / 10 / 2119: 00$ \\
$2019 / 10 / 2119: 00$ & $2019 / 11 / 112: 00$ \\
$2019 / 11 / 112: 00$ & $2019 / 11 / 1117: 00$ \\
$2019 / 11 / 1117: 00$ & $2019 / 11 / 2213: 00$ \\
$2019 / 11 / 2213: 00$ & $2019 / 12 / 217: 00$ \\
$2019 / 12 / 217: 00$ & $2019 / 12 / 1116: 00$ \\
$2019 / 12 / 1116: 00$ & $2019 / 12 / 2117: 50$ \\
$2019 / 12 / 2117: 50$ & $2020 / 1 / 217: 50$ \\
$2020 / 1 / 217: 50$ & $2020 / 1 / 1211: 50$ \\
$2020 / 1 / 1211: 50$ & $2020 / 1 / 2116: 50$ \\
$2020 / 1 / 2116: 50$ & $2020 / 2 / 19: 50$ \\
$2020 / 2 / 19: 50$ & $2020 / 2 / 1216: 50$ \\
$2020 / 2 / 1216: 50$ & $2020 / 2 / 2114: 50$ \\
$2020 / 2 / 2114: 50$ & $2020 / 3 / 114: 50$ \\
$2020 / 3 / 114: 50$ & $2020 / 3 / 1114: 50$ \\
$2020 / 3 / 1114: 50$ & $2020 / 3 / 2117: 50$ \\
2020/3/21 17:50 & $2020 / 3 / 3118: 50$ \\
\hline & \\
&
\end{tabular}


Table S2 Statistics metrics of species Hg, meteorological factors and other pollutants

\begin{tabular}{|c|c|c|c|c|c|c|c|c|c|c|c|c|c|}
\hline Period & Statistics & $\begin{array}{c}\text { GEM } \\
\left(\mathrm{ng} \mathrm{m}^{-3}\right)\end{array}$ & $\begin{array}{c}\text { PBM } \\
\left(\mathrm{pg} \mathrm{m}^{-3}\right)\end{array}$ & $\begin{array}{c}\text { GOM } \\
\left(\mathrm{pg} \mathrm{m}^{-3}\right)\end{array}$ & $\begin{array}{l}\text { Temp } \\
\left({ }^{\circ} \mathrm{C}\right)\end{array}$ & $\begin{array}{l}\text { Hum } \\
(\%)\end{array}$ & $\begin{array}{c}\text { Wind_Speed } \\
\qquad\left(\mathrm{m} \mathrm{s}^{-1}\right)\end{array}$ & $\begin{array}{c}\text { Solar_Rad. } \\
\left(\mathrm{W} \mathrm{m}^{-2}\right)\end{array}$ & $\begin{array}{c}\mathrm{CO} \\
\left(\mathrm{mg} \mathrm{m}^{-3}\right)\end{array}$ & $\begin{array}{c}\mathrm{NO}_{2} \\
\left(\mu \mathrm{g} \mathrm{m}^{-3}\right)\end{array}$ & $\begin{array}{c}\mathrm{O}_{3} \\
\left(\mu \mathrm{g} \mathrm{m}^{-3}\right)\end{array}$ & $\begin{array}{c}\mathrm{PM}_{2.5} \\
\left(\mu \mathrm{g} \mathrm{m}^{-3}\right)\end{array}$ & $\begin{array}{c}\mathrm{SO}_{2} \\
\left(\mu \mathrm{g} \mathrm{m}^{-3}\right)\end{array}$ \\
\hline \multirow{5}{*}{ PISM } & mean & 1.2 & 11.4 & 13.5 & 6.05 & 72.63 & 0.61 & 182.35 & 0.46 & 6.77 & 86.5 & 8.69 & 3.76 \\
\hline & sd & 0.35 & 4.8 & 7.3 & 5.46 & 22 & 1.04 & 282.68 & 0.18 & 3.2 & 22.68 & 4.42 & 2.88 \\
\hline & median & 1.17 & 10.9 & 13.6 & 5.8 & 82 & 0 & 11 & 0.45 & 6 & 88 & 8 & 3 \\
\hline & $\min$ & 0.5 & 0.3 & 0.6 & -9.3 & 14 & 0 & 0 & 0.2 & 3 & 20 & 1 & 1 \\
\hline & $\max$ & 5.13 & 24.6 & 48.1 & 20.7 & 96 & 6.3 & 1346 & 3.8 & 24.5 & 156 & 59 & 64.5 \\
\hline \multirow{5}{*}{ ISM1 } & mean & 0.92 & 15.4 & 18.2 & 8.6 & 81.85 & 0.69 & 162.79 & 0.38 & 7.19 & 87.27 & 11.31 & 7.54 \\
\hline & sd & 0.23 & 7.9 & 29.2 & 3.5 & 15.14 & 1.09 & 248.07 & 0.07 & 2.94 & 23.26 & 7.63 & 0.9 \\
\hline & median & 0.9 & 15.1 & 10.9 & 7.9 & 88 & 0 & 25 & 0.4 & 6 & 88.5 & 9.5 & 7.5 \\
\hline & $\min$ & 0.5 & 0.4 & 0.9 & -2.2 & 16 & 0 & 0 & 0.2 & 3 & 26 & 1 & 6 \\
\hline & $\max$ & 2.59 & 50.2 & 270.2 & 20.2 & 96 & 6.7 & 1502 & 0.6 & 22 & 147.5 & 55 & 27 \\
\hline \multirow{5}{*}{ ISM2 } & mean & 0.92 & 7.9 & 13.5 & 12.9 & 85.7 & 0.29 & 164.7 & 0.44 & 7.38 & 55.27 & 6.62 & 8.61 \\
\hline & $\mathrm{sd}$ & 0.18 & 3.4 & 5.5 & 3.1 & 12.56 & 0.73 & 246.89 & 0.06 & 2.72 & 25.58 & 1.84 & 1.75 \\
\hline & median & 0.91 & 7.4 & 12.9 & 12.2 & 92 & 0 & 28 & 0.45 & 6.5 & 52 & 6.5 & 8.5 \\
\hline & $\min$ & 0.5 & 1.3 & 3.6 & -0.1 & 36 & 0 & 0 & 0.3 & 3 & 6 & 3 & 5 \\
\hline & $\max$ & 2.1 & 29.3 & 105.0 & 23.9 & 97 & 6.7 & 1503 & 0.7 & 19.5 & 136.5 & 19 & 38.5 \\
\hline \multirow{5}{*}{ ISM3 } & mean & 1.04 & 3.9 & 6.0 & 13.97 & 80.71 & 0.17 & 199.79 & 0.36 & 6.71 & 50.34 & 6.58 & 7.21 \\
\hline & $\mathrm{sd}$ & 0.21 & 3.6 & 5.0 & 4.43 & 16.52 & 0.54 & 298.95 & 0.06 & 2.53 & 22.26 & 2.05 & 4.06 \\
\hline & median & 1.03 & 2.4 & 4.4 & 12.7 & 89 & 0 & 14 & 0.35 & 6 & 49.5 & 6.5 & 8 \\
\hline & $\min$ & 0.52 & 0.01 & 0.03 & 3.8 & 27 & 0 & 0 & 0.25 & 4 & 6.5 & 1.5 & 4 \\
\hline & $\max$ & 1.82 & 16.1 & 25.7 & 27.7 & 97 & 5.4 & 1394 & 0.55 & 20.5 & 114 & 23.5 & 75.5 \\
\hline
\end{tabular}


Table S3 daily average concentrations of $\mathrm{Hg}$ species, meteorological and some pollutants from 31 May to 3 June, 2019. Red markers indicate large changes in factors

\begin{tabular}{cc|cccc}
\hline & & May 31 & June 1st & June 2nd & June 3rd \\
\hline GEM & $\left(\mathrm{ng} \mathrm{m}^{-3}\right)$ & 0.96 & 1.18 & 1.03 & 1.07 \\
GOM & $\left(\mathrm{pg} \mathrm{m}^{-3}\right)$ & 14.6 & 106.1 & 40.6 & 34.6 \\
PBM & $\left(\mathrm{pg} \mathrm{m}^{-3}\right)$ & 25.4 & 11.5 & 18.8 & 23 \\
Temp & $\left({ }^{\circ} \mathrm{C}\right)$ & 8.64 & 10.30 & 9.47 & 9.86 \\
Hum & $(\%)$ & 76.60 & 58.17 & 58.95 & 67.38 \\
WS & $\left(\mathrm{m} \mathrm{s}^{-1}\right)$ & 0.48 & 0.71 & 1.22 & 1.05 \\
Bar & $\left(\mathrm{hPa}^{2}\right.$ & 680.93 & 680.57 & 681.28 & 678.84 \\
Rain & $\left(\mathrm{mm}^{2}\right)$ & 0.00 & 0.00 & 0.00 & 0.00 \\
Solar_Rad. & $\left(\mathrm{W} \mathrm{m}^{-2}\right)$ & 189.24 & 324.49 & 300.11 & 207.99 \\
UV Index & - & 1.30 & 2.01 & 1.93 & 1.36 \\
$\mathrm{CO}$ & $\left(\mathrm{mg} \mathrm{m}^{-3}\right)$ & 0.46 & 0.40 & 0.41 & 0.43 \\
$\mathrm{NO}_{2}$ & $\left(\mu \mathrm{g} \mathrm{m}^{-3}\right)$ & 7.80 & 7.83 & 8.34 & 8.45 \\
$\mathrm{O}_{3}$ & $\left(\mu \mathrm{g} \mathrm{m}^{-3}\right)$ & 105.33 & 94.40 & 100.61 & 106.00 \\
$\mathrm{PM}_{10}$ & $\left(\mu \mathrm{g} \mathrm{m}^{-3}\right)$ & 12.45 & 14.25 & 24.47 & 20.00 \\
$\mathrm{PM}_{2.5}$ & $\left(\mu \mathrm{g} \mathrm{m}^{-3}\right)$ & 9.48 & 11.33 & 12.05 & 10.05 \\
$\mathrm{SO}_{2}$ & $\left(\mu \mathrm{g} \mathrm{m}^{-3}\right)$ & 8.13 & 8.02 & 8.28 & 8.25 \\
\hline
\end{tabular}

Note: Red markers indicate large changes in factors 